\title{
Assessment of Midfacial Hypoplasia in Down Syndrome Fetuses - Validity of a Two-Line Approach and Introduction of a Novel Angle (Maxilla-Mandible- Nasion Angle)
}

\author{
Authors \\ J. Weichert ${ }^{1}$, M. Gembicki ${ }^{1}$, J. Ribbat-Idel ${ }^{2}$, D. R. Hartge \\ Affiliations \\ ${ }^{1}$ Prenatal Medicine, University Hospital of Schleswig-Holstein, Luebeck, Germany \\ ${ }^{2}$ Department of Pathology, University Hospital of Schleswig-Holstein, Luebeck, Germany
}

Key words
face
jaws
midfacial hypoplasia
trisomy 21
3D ultrasound

received $\quad 04.08 .2015$

accepted 22.03.2016

Bibliography

DOI http://dx.doi.org/

10.1055/s-0042-106397

Ultrasound International Open

2016; 2: E58-E62

(c) Georg Thieme Verlag KG

Stuttgart · New York

ISSN 2199-7152

Correspondence

Dr. J. Weichert

Prenatal Medicine

University Hospital of

Schleswig-Holstein

Ratzeburger Allee 160

23538 Luebeck

Germany

Tel.: + 49/451/5002 149

Fax: +49/451/5002 192

jan.weichert@uksh.de



\begin{abstract}
$\nabla$

Purpose: To scrutinize the validity of a novel angle (maxilla-mandible-nasion angle, MMN) as objective proof of midfacial hypoplasia in trisomy 21 fetuses.

Materials and Methods: Volume data sets of $2^{\text {nd }}$ trimester fetuses were reviewed in this retrospective study. After achievement of the correct midsagittal position, the fetal profile line (FP line) and the mandibulo-maxillary line (MML) were applied and the resulting angle was calculated. Additionally, the prefrontal space ratio (PFSR) was assessed. Both measurements were obtained from 401 euploid fetuses and 42 fetuses with trisomy 21 . Values for MMN and PFSR $<5^{\text {th }}$ percentile were considered abnormal.
\end{abstract}

\section{Introduction}

Nearly 40 years ago, Westerman analyzed palatal casts of individuals with Down syndrome (DS) and described the palatal dimensions as markedly narrower in width, shorter in depth and lower in height than in normal controls [1]. The mean maxillary depth as a measure of midfacial hypoplasia was significantly smaller in 3D volume data sets of first-trimester fetuses with trisomy 21 compared to normal [2]. Accordingly, Alió et al. also reported maxillary hypoplasia of about $10 \mathrm{~mm}$ in children with Down syndrome compared to controls [3]. Beside a shortened maxilla, an absent vomer has recently been described in first- and second-trimester fetuses, thus emphasizing midfacial hypoplasia as a consistent finding in trisomy 21 [4]. Hypo- or oligodontia found in up to $60 \%$ of patients with DS [5] constitutes another frequent abnormality, which is in line with the finding of an underdevelopment of the upper jaw compared to the mandibular growth [6]. Farkas et al. provided further evi-
Results: The study included 443 fetuses with a mean gestational age of 21.3 weeks (range: $14.0-$ 26.3). The MMN angle sufficiently identified hypoplasia of the midface in trisomy 21 fetuses (mean: $14.6^{\circ}$; range: $10.1^{\circ}-22.0^{\circ}$ ) compared to controls (mean: $20.5^{\circ}$; range: $17.3^{\circ}-23.7^{\circ}$; $\mathrm{p}<0.0001$ ). Concomitantly, the PFSR of Down syndrome fetuses was significantly lower (mean: 0.53 ; range: $0.21-1.22$ ) than in euploid individuals (1.38; range: $0.54-2.23 ; \mathrm{p}<0.0001$ ).

Conclusion: Calculation of the novel MMN angle in $2^{\text {nd }}$ trimester fetuses reliably allows rapid assessment of craniofacial anatomy in order to rule out the midfacial hypoplasia frequently found in trisomy 21.

dence for an abnormal craniofacial surface anatomy in trisomy 21 patients assessed by linear and angular measurements [7].

In the recent past a growing number of different angles have been introduced, all developed for an exact and reproducible assessment of craniofacial integrity. With regard to prenatal identification of trisomy 21 fetuses, application of the frontomaxillary angle [8,9] and the recently introduced calculation of the prefrontal space ratio (PFSR) are of diagnostic value [10]. De JongPleij et al. established the fetal profile line (FPL) drawn from the nasion to the mandible in order to get information about forehead anomalies and/or anomalies of the maxilla-mandible complex [11].

In this study we examined the applicability and clinical value of a novel angle combining 2 lines: the fetal profile line and the maxilla-mandibular line (essential for assessment of the PFSR) in order to obtain information about craniofacial architecture and abnormalities and the use of angle calculation for aiding the diagnosis of aneuploidy in $2^{\text {nd }}$ trimester pregnancies. 


\section{Methods}

\section{$\nabla$}

The protocol for this retrospective study (11/2009-2-2/2014) was approved by the local ethics committee and all patients enrolled in this analysis gave informed consent. Stored threedimensional (3D) volume data sets were reviewed, all being obtained transabdominally in second-trimester fetuses referred for targeted ultrasound examination with Voluson E8 expert and 730 expert systems (GE Healthcare, Zipf, Austria) equipped with a RM6C matrix and RAB4-8L convex probe, respectively. All sonographic examinations were performed by a single experienced operator. The volumes were acquired with the fetus facing the transducer. The 3D sweep acquisition angle ranged from $45-55^{\circ}$ to ensure depiction of the entire face. After volume adjustment for an exact midsagittal section approved in corresponding transverse and coronal sections (multiplanar mode) and subsequent magnification, all included profiles were assessed with the fetal head in a vertical position (no flexion or extension and the mouth closed in order to prevent displacement of the mandible) as displayed in $\bullet$ Fig. 1. The anatomical landmarks essential for angle calculation were the nasion (defined as the intersection of the frontal and nasal bones) and the anteriormost points of both the mandible and the maxilla. Calipers were placed and 2 lines were drawn connecting the ventral edge of the mandible with the nasion (fetal profile line; FPL) and a second one from the mandible to the anterior edge of the maxilla as recently described (mandibulo-maxillary line; MML). The resulting angle was introduced as the maxilla-mandible-nasion angle (MMN, $\bullet$ Fig. 1,2). Additionally, we calcu- lated the prefrontal space ratio (PFSR) by measuring the distance between the fetal profile line traversing the nasion (at the level of the nasion) and the leading edge of the frontal skin and a second one between the skin and the interception with the MML as introduced by Sonek et al. [10]. The PFSR was subsequently estimated by division of the latter by the distance between the FPL and skin. All MMN values assessed in trisomy 21 fetuses were compared to those derived from normal controls (healthy fetuses with known perinatal outcome).

\section{Statistical analysis}

Mann-Whitney $U$-test for independent samples was used for comparison of the mean MMN and PFSR values measured in normal controls and trisomy 21 fetuses. Correlation was assessed using Pearson's correlation test. The data were analyzed using the statistical software SPSS 21.0 (Chicago, IL) and Microsoft Excel 2010 (Microsoft Corp, Redmond, WA). P-values of less than 0.05 were considered statistically significant.

\section{Results}

$\nabla$

A total of 471 volume data sets were reviewed. The mean gestational age was 21.3 weeks (ranging from 14.0 to 26.3 gestational weeks) with no significant difference between the control and the trisomy 21 group. The mean maternal age was slightly higher in the trisomy 21 group (35.9 years; ranging from 21 to 48 years) than in the control group (32.4 years; ranging from 16 to 50 years). All women enrolled in this study were of Caucasian
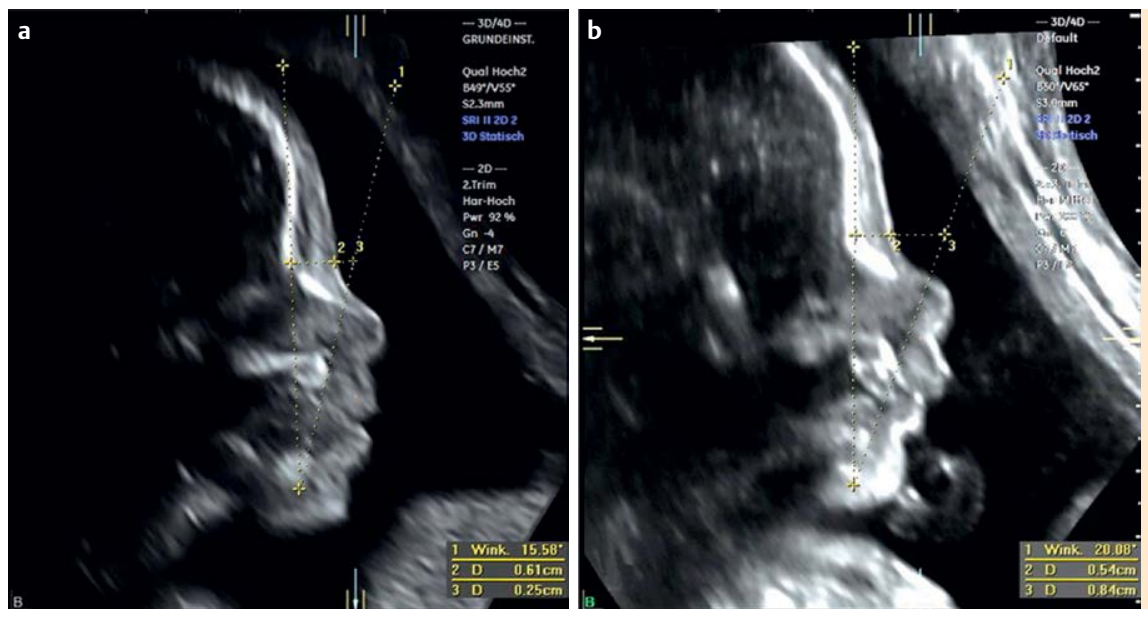

Fig. 1 Fetal profiles obtained from facial volumes of a trisomy 21 fetus $\mathbf{a}$ and an euploid fetus $\mathbf{b}$ at 22 weeks of gestation. Left panel corresponds to the coronal view with the reference dot placed between the nostrils. Sagittal plane showing 2 lines tracing the mandible, the nasion and the anteriormost border of the maxilla, respectively. The resulting novel $\mathrm{MMN}$ angle is significantly more acute in a Down syndrome fetus. In addition, the PFSR is markedly decreased (panel a) in this fetus compared to controls (panel b).
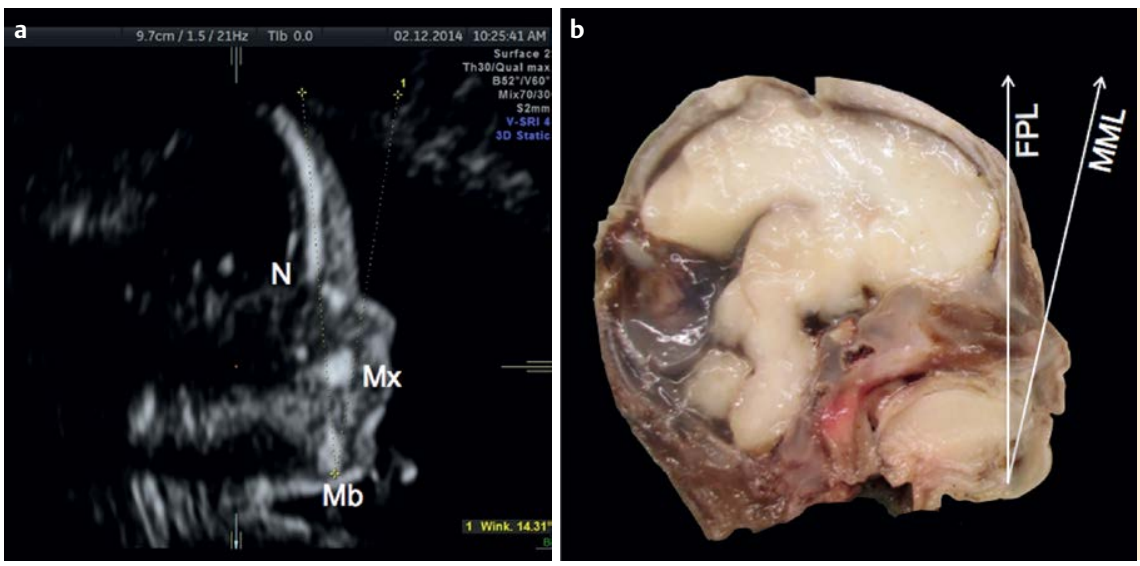

Fig. 2 Midsagittal view of a Down syndrome fetus at 21 gestational weeks demonstrating an acute MMN $<5^{\text {th }}$ percentile (panel a). Macroscopic specimen of the same fetus after TOP clearly depicting anatomical landmarks for assessing midfacial hypoplasia (panel b). FPL: fetal profile line; $\mathrm{N}$ : nasion; Mb: mandible; MML: mandibulo-maxillary line; $M x$ : maxilla. 

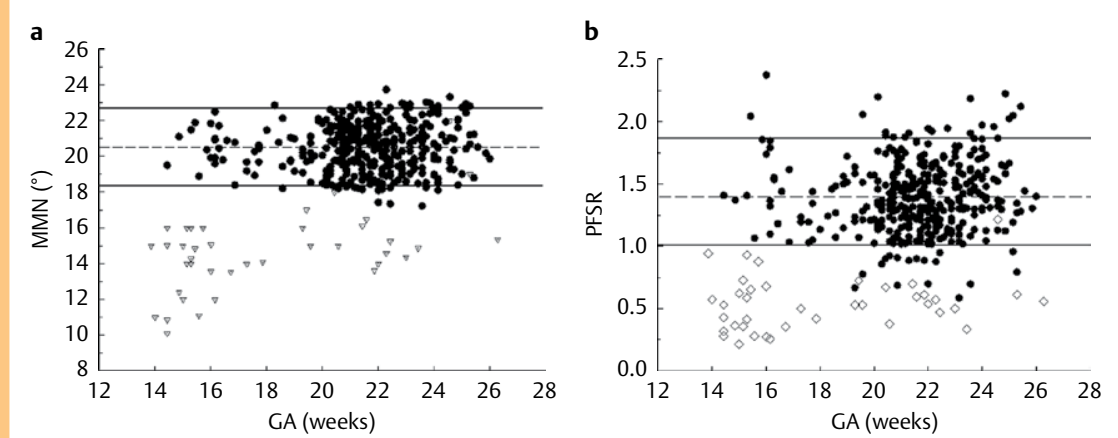

Fig. 3 Scatterplot of maxilla-mandibula-nasion angle (MMN; panel a) and prefrontal space ratio (PFSR; panel b) measurements in normal controls and trisomy 21 fetuses. Solid lines represent the $5^{\text {th }}$ and $95^{\text {th }}$ percentile, and broken line shows mean values.
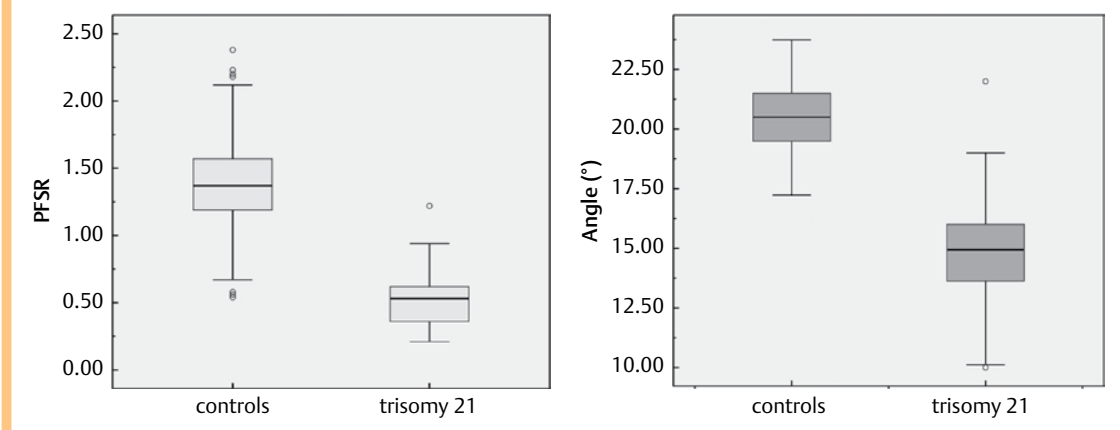

Fig. 4 Boxplots of (MMN; panel a) and (PFSR; panel b) in normal and trisomy 21 fetuses. Boxes represent the median, $25^{\text {th }}$ and $75^{\text {th }}$ percentiles. Whiskers show the range (minimum and maximum). Circles represent outliers.

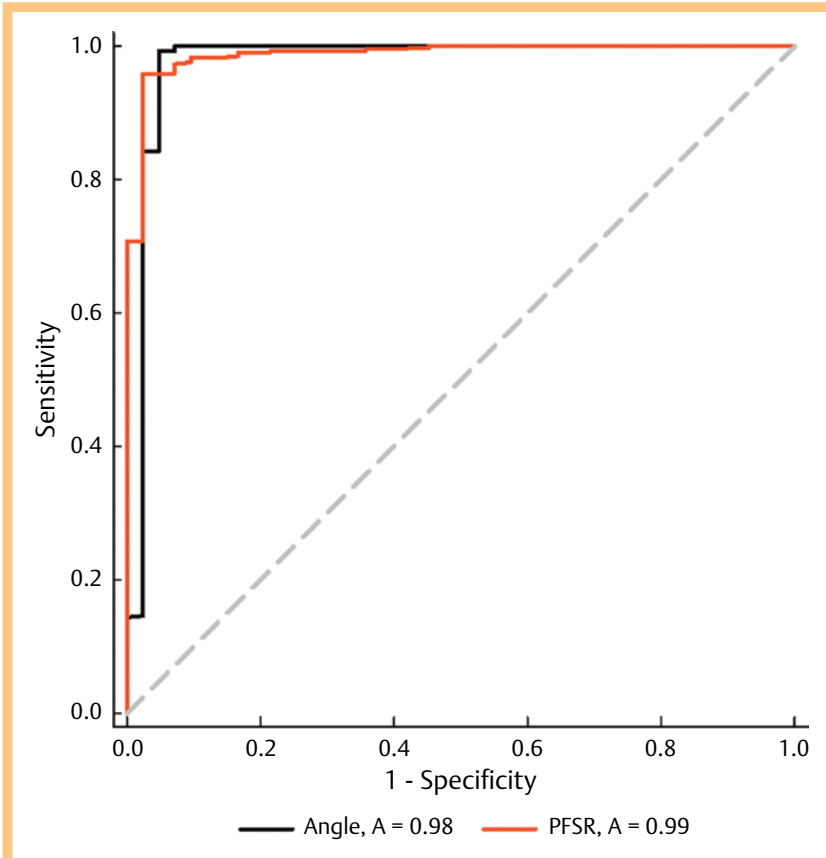

Fig. 5 Receiver operating characteristic analysis of MMN and PFSR in 2 nd trimester fetuses. The area under the ROC curve for identifying trisomy 21 fetuses was 0.976 using MMN and 0.987 when assessing the PFSR.

ethnicity. 28 cases were excluded because of other structural or genetic anomalies or had an initial acquisition plane too far from the true midsagittal plane, leaving 443 eligible cases for final analyses. Of those, 42 fetuses had trisomy 21 confirmed by antenatal karyotyping. The remaining 401 patients had a normal appearance during targeted ultrasound, with 19 cases $(4.7 \%)$ being found to have a positive fetal profile line (FPL passing the frontal bone posteriorly). In contrast, none of the Down syndrome fetuses presented with forehead anomalies. Neither in euploid controls nor in trisomy 21 fetuses could a negative FPL (position anterior to the frontal bone) be demonstrated.

Correct identification of anatomical landmarks enabled sufficient angle calculation in all study cases enrolled in the final assessment. The MMN angle in normal controls was $20.5^{\circ}$ (range from $17.3^{\circ}$ to $23.7^{\circ}$ ), whereas this measurement was significantly more acute (mean: $14.6^{\circ}$; range: $10.1^{\circ}$ to $22.0^{\circ}$ ) in cases with trisomy 21 as shown in ( $\bullet$ Fig. 3,4$)(p<0.0001)$. The 5 th and $95^{\text {th }}$ percentiles were $18.4^{\circ}$ and $22.7^{\circ}$, respectively. The prefrontal space ratio could readily be calculated in both groups, showing that the PFSR in trisomy 21 cases was significantly smaller (mean: 0.53; range: 0.21-1.22) compared to euploid fetuses who had a mean PFSR of 1.38, ranging from 0.54 to 2.23 $(p<0.0001)$. The 5 th and 95th percentiles for PFSR in our cohort were 1.02 and 1.89 , respectively. In 2 fetuses with trisomy 21 a normal nasal bone length ( $>5^{\text {th }}$ percentile) could be noticed, and both had a normal PFSR ( $>5^{\text {th }}$ percentile). In $18 / 401$ normal fetuses $(4.5 \%)$, the PFSR was $<1$, and in another $11 / 401$ cases $(2.7 \%)$ the MMN was below the $5^{\text {th }}$ percentile.

Receiver operating characteristic curves (ROC) were used to emphasize the predictive accuracy of angle assessment and calculation of the PFSR for the detection of trisomy 21. The AUC for both variables were 0.976 (MMN) and 0.987 (PFSR), respectively (๑ Fig. 5).

In euploid fetuses we found no correlation between gestational age and the MMN angle (Pearson $r=0.06, P=0.12$ ) and PFSR $(r=0.45, P=0.20)$. A slight increase in MMN values with advancing GA in Down syndrome fetuses was observed (Pearson $r=0.27, P=0.04$ ) exclusively when assessed in early second trimester. Again, no correlation between PFSR and GA in trisomy 21 cases could be demonstrated $(r=-0.02, P=0.46)$. 


\section{Discussion}

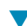

In our study on 3D volume data sets of mid-trimester fetuses, we were able to demonstrate significant differences in craniofacial anatomy in euploid and trisomy 21 fetuses. These changes in craniofacial architecture of individuals with Down syndrome are confined to midfacial hypoplasia. However, there are other changes that often are noted postnatally as well, such as narrow nasal airways, macroglossia, glossoptosis, tonsil and adenoid hypertrophy, and increased collapsibility of the upper airway $[12,13]$. In fact, hypoplasia of the midface in addition to other well-described structural markers has been confirmed to be a common prenatal feature of trisomy 21 fetuses. The main morphological determinant is a hypoplastic and dorsally retracted maxilla present as early as the first trimester [2,14,15]. Based on both the dorsal displacement of the maxilla and the observation that prenasal thickness is above the $95^{\text {th }}$ percentile in $>70 \%$ of mid-trimester Down syndrome fetuses [16], the prefrontal space ratio (PFSR) has been introduced as a highly effective tool incorporating both variables [10]. In this regard the predictive value of another marker for trisomy 21, namely the prenasal thickness-to-nasal bone length ratio (PT-NBL), has recently been confirmed in high-risk $[17,18]$ and low-risk prenatal populations [19].

The novel maxilla-mandible-nasion angle (MMN) proved to be a valid and useful tool in the rapid assessment of craniofacial integrity as this measure yields valuable information taken from 2 independent reference lines (FPL and MML).

As our data suggest, an acute MMN (mean: $14.6^{\circ}, \mathrm{SD}: 2.30$ ) appears to be predictive of midfacial hypoplasia found in $41 / 42$ trisomy 21 fetuses compared to normal fetuses having a mean MMN value of 20.5 (SD: 1.33). The significantly lower PFSR in Down syndrome fetuses (mean: 0.53; SD: 0.21) compared to euploid controls (mean: 1.38 , SD: 0.29 ) in our study is consistent with the observations made in previous studies using either 3D volumes [10] or 2D images [20]. According to recent data, we were able to reproduce the robustness of the PFSR in Down syndrome risk assessment irrespective of gestational age [21]. Despite a slight increase of MMN in trisomic fetuses during the early second trimester, this measurement remained stable below the $5^{\text {th }}$ percentile in all affected fetuses.

As previously published, the FPL is reported to be always zero in euploid fetuses (FPL traverses the frontal bone lengthwise) until 27 gestational weeks. It was therefore reasonable to implement this line in PFSR calculation as described. Recent data provided by Vos et al. suggest that in second and particularly in third trimester Down syndrome fetuses a positive FPL could be noticed [22]. However, in our study none of the trisomy 21 fetuses had forehead anomalies corresponding to a positive FPL. Yazdi et al. found that the MML coursed behind the prenasal skin in a considerable number of Down syndrome cases which is potentially predictive of fetal aneuploidy in the second and third trimester [20]. Recently, the same group reported significant differences between euploid and aneuploid fetuses (including trisomy 21, 18 and 13) regarding the frontal space assessed at 11-13 gestational weeks and the impact on first-trimester combined aneuploidy screening [23]. In contrast to the described MMN angle having its vertex on the anterior edge of the mandible, the previously described MNM (between 2 lines originating from the nasion and tracing the anterior borders of the mandible and the maxilla, respectively) [24] is found to be a weak DS marker as less than $25 \%$ of trisomy 21 fetuses had abnormal values [22].
There have been a number of publications dealing with the potential superiority of 3D vs. 2D-based studies on facial integrity in normal and aneuploid fetuses [25-28]. It has been shown that the facial maxillary angle is significantly larger when assessed via a three-dimensional approach. Similarly, nasal bone length measured in an exact midsagittal plane is proven to be significantly smaller [28]. Recently, a study on 105 fetuses demonstrated that the maxilla-nasion-mandible angle (MNM) calculated on 2D images was significantly larger than on 3D images [29]. In terms of the ability to sufficiently discriminate between euploid and Down syndrome fetuses, neither assessment of the MNM angle nor all other markers analyzed in this study revealed statistically significant differences when measured on two- or three-dimensionally acquired images (data not shown). However, three-dimensional multiplanar confirmation of a true midsagittal section was thought to be mandatory in our study in order to provide precise and reliable values of MMN and to preclude parasagittal shifting.

The study has several limitations including the retrospective design and the fact that the measurements were made by a single operator not blinded to the fetal karyotype.

In conclusion, the present study demonstrates that combining the advantages of both the FPL and the mandibulo-maxillary line using identical midsagittal sonographic planes of the fetal profile offers the opportunity to rapidly detect $2^{\text {nd }}$ trimester fetuses (from 14 completed weeks onwards) at risk for trisomy 21 (assessed by the novel MMN angle). Regardless of the recent advances in noninvasive prenatal testing utilizing cell-free fetal DNA, this is still an important issue during targeted ultrasound and subsequent prenatal counseling [30].

The reliability of MMN angle assessment to identify other fetal conditions influencing craniofacial integrity (e.g., micrognathia, facial clefting) has to be established in future studies.

\section{References}

1 Westerman GH, Johnson R, Cohen MM. Variations of palatal dimensions in patients with Down's syndrome. J Dent Res 1975; 54: 767-771

2 Dagklis $T$, Borenstein $M$, Peralta CF et al. Three-dimensional evaluation of mid-facial hypoplasia in fetuses with trisomy 21 at $11+0$ to $13+6$ weeks of gestation. Ultrasound Obstet Gynecol 2006; 28: 261-265

3 Alió J, Lorenzo J, Iglesias MC et al. Longitudinal maxillary growth in Down syndrome patients. Angle Orthod 2011; 81: 253-259

4 Mihailovic T, Stimec BV, Terzic M et al. The absence of the vomer in the first and early second trimester of pregnancy - a new marker of trisomy 21 and trisomy 13. Ultraschall Med 2012; 33: E68-E74

5 Andersson EM, Axelsson S, Austeng ME et al. Bilateral hypodontia is more common than unilateral hypodontia in children with Down syndrome: a prospective population-based study. Eur J Orthod 2014; 36: 414-418

6 Allanson JE, O'Hara P, Farkas LG et al. Anthropometric craniofacial pattern profiles in Down syndrome. Am J Med Genet 1993; 47: 748-752

7 Farkas LG, Katic MJ, Forrest CR et al. Surface anatomy of the face in Down's syndrome: linear and angular measurements in the craniofacial regions. J Craniofac Surg 2001; 12: 373-379

8 Sonek J, Borenstein M, Dagklis T et al. Frontomaxillary facial angle in fetuses with trisomy 21 at 11-13(6) weeks. Am J Obstet Gynecol 2007; 196: e1-e4

9 Molina $F$, Persico $N$, Borenstein $M$ et al. Frontomaxillary facial angle in trisomy 21 fetuses at 16-24 weeks of gestation. Ultrasound Obstet Gynecol 2008; 31: 384-387

10 Sonek J, Molina F, Hiett AK et al. Prefrontal space ratio: comparison between trisomy 21 and euploid fetuses in the second trimester. Ultrasound Obstet Gynecol 2012; 4: 293-296

11 de Jong-Pleij EA, Ribbert LS, Pistorius $L R$ et al. The fetal profile line: a proposal for a sonographic reference line to classify forehead and mandible anomalies in the second and third trimester. Prenat Diagn 2012; 32: 797-802 
12 Ferrario VF, Dellavia C, Zanotti G et al. Soft tissue facial anthropometry in Down syndrome subjects. J Craniofac Surg 2004; 15: 528-532

13 Suri S, Tompson BD, Cornfoot $L$. Cranial base, maxillary and mandibular morphology in Down syndrome. Angle Orthod 2010; 80: 861-869

14 Cicero S, Curcio P, Rembouskos $G$ et al. Maxillary length at 11-14 weeks of gestation in fetuses with trisomy 21. Ultrasound Obstet Gynecol 2004; 24: 19-22

15 Sforza C, Dellavia C, Dolci $C$ et al. A quantitative three-dimensional assessment of abnormal variations in the facial soft tissues of individuals with Down syndrome. Cleft Palate Craniofac J 2005; 42: 410-416

16 Persico N, Borenstein M, Molina Fet al. Prenasal thickness in trisomy-21 fetuses at 16-24 weeks of gestation. Ultrasound Obstet Gynecol 2008; 32: 751-754

17 de Jong-Pleij EA, Vos FI, Ribbert LS et al. Prenasal thickness-to-nasal bone length ratio: a strong and simple second- and third-trimester marker for trisomy 21. Ultrasound Obstet Gynecol 2012; 39: 185-190

18 Vos FI, De Jong-Pleij EA, Bakker M et al. Trends in serial measurements of ultrasound markers in second and third trimester Down syndrome fetuses. Fetal Diagn Ther 2015 Jan 30. [Epub ahead of print]

19 Gonzalez R, Aedo S, Dezerega $V$ et al. Frontonasal fold thickness-tonasal bone length ratio as a prenatal sonographic marker for trisomy 21 in a low-risk population. J Ultrasound Med 2013; 32: 795-800

20 Yazdi B, Sonek J, Oettling $C$ et al. Prefrontal space ratio in second- and third-trimester screening for trisomy 21. Ultrasound Obstet Gynecol 2013; 41: 262-266

21 Chaveeva P, Agathokleous M, Poon LC et al. Second-trimester screening for trisomy-21 using prefrontal space ratio. Fetal Diagn Ther 2013; 34: $50-55$

22 Vos FI, de Jong-Pleij EA, Bakker $M$ et al. Nasal bone length, prenasal thickness, prenasal thickness to nasal bone length ratio and the prefrontal space ratio in second and third trimester Down syndrome. Ultrasound Obstet Gynecol 2014, Apr 21 doi:10.1002/uog.13391 [Epub ahead of print]
23 Yazdi B, Riefler P, Fischmüller K et al. The frontal space measurement in euploid and aneuploid pregnancies at 11-13 weeks' gestation. Prenat Diagn 2013; 33: 1124-1130

24 de Jong-Pleij EA, Ribbert LS, Manten GT et al. Maxilla-nasion-mandible angle: a new method to assess profile anomalies in pregnancy. Ultrasound Obstet Gynecol 2011; 37: 562-569

25 Alphonse J, Cox J, Clarke J et al. Comparison of frontomaxillary facial angles using both 2D and 3D ultrasound at $11+0$ to $13+6$ weeks of gestation. Fetal Diagn Ther 2010; 28: 14-21

26 de Jong-Pleij EA, Ribbert LS, Tromp E et al. Three-dimensional multiplanar ultrasound is a valuable tool in the study of the fetal profile in the second trimester of pregnancy. Ultrasound Obstet Gynecol 2010; 35: $195-200$

27 Ramos GA, Kfir M, Lee $S$ et al. Benefits of a systematic approach in the evaluation of fetal facial 3-dimensional volumes. J Ultrasound Med 2011; 30: 473-479

28 Vos FI, de Jong-Pleij EA, Ribbert LS et al. Three-dimensional ultrasound imaging and measurement of nasal bone length, prenasal thickness and frontomaxillary facial angle in normal second- and third-trimester fetuses. Ultrasound Obstet Gynecol 2012; 39: 636-641

29 Vos FI, Bakker M, de Jong-Pleij EA, Ribbert LS et al. Is 3D technique superior to 2D in Down syndrome screening? Evaluation of six second and third trimester fetal profile markers. Prenat Diagn 2014, Oct 23 doi:10.1002/pd.4521 [Epub ahead of print]

30 Kagan KO, Hoopmann M, Hammer $R$ et al. Screening auf Chromosomenstörungen mittels Ersttrimester-Screening und non-invasive prenatal Testing. Ultraschall Med 2015; 36: 40-46 